\title{
ON BILINEAR KINETIC EQUATIONS. BETWEEN MICRO AND MACRO DESCRIPTIONS OF BIOLOGICAL POPULATIONS
}

\author{
MIROSŁAW LACHOWICZ \\ Institute of Applied Mathematics and Mechanics \\ Faculty of Mathematics, Informatics and Mechanics, Warsaw University \\ Banacha 2, 02-097 Warszawa, Poland \\ E-mail:lachowic@mimuw.edu.pl
}

\begin{abstract}
In this paper a general class of Boltzmann-like bilinear integro-differential systems of equations (GKM, Generalized Kinetic Models) is considered. It is shown that their solutions can be approximated by the solutions of appropriate systems describing the dynamics of individuals undergoing stochastic interactions (at the "microscopic level"). The rate of approximation can be controlled. On the other hand the GKM result in various models known in biomathematics (at the "macroscopic level") including the "SIR" model, some competitive systems and the Smoluchowski coagulation model.
\end{abstract}

1. Introduction. Usually the description of biological populations is carried out on macroscopic level of interacting subpopulations of the system. However in many cases the description on microscopic level of interacting individuals (e.g. cells) seems to be more adequate.

For example the evolution of a neoplastic cell (see e.g. [He], [BD]), can be described according to the following steps:

1. loss of differentiation and replication: the cells start to reproduce in the form of identical descendants (so-called clonal expansion);

2000 Mathematics Subject Classification: 35K45, 45K05, 82A40, 92D25, 76P05.

Key words and phrases: integro-differential equations, population dynamics, stochastic particle systems, propagation of chaos.

The present work was produced within the framework of EC Programme "Improving the Human Research Potential and Socio-Economic Knowledge Base"-Research Training Networks, Con. N. HPRN-CT-2000-00105, "Using Mathematical Modelling and Computer Simulation to Improve Cancer Therapy". The work bases on the author's lecture at the Conference on Mathematical Modelling of Population Dynamics, Będlewo, June 24-28, 2002.

The paper is in final form and no version of it will be published elsewhere. 
2. interaction (activation or inhibition) and competition at the cellular level with immune and environmental cells, e.g. through the emission of cytokine signals;

3. condensation of tumour cells, macroscopic diffusion and angiogenesis;

4. detachment of metastases and propagation.

The first two steps imply cellular and sub-cellular interactions, while the last two can be described at the macroscopic level.

Mathematical models of cellular phenomena related to the interaction between tumour cells and the immune system have been developed by Bellomo and Forni $[\mathrm{BF}]$ by methods which are typical of kinetic theory. Bellomo and Forni's model is a generalization of the Jäger and Segel [JS] kinetic model.

In $[\mathrm{ABL}]$ a class of models of population dynamics with kinetic interactions taking into account transitions among different populations was proposed. Further generalization of the class [ABL] was proposed and analysed in [LW2] (see also [La4] and references therein). This class includes, as particular cases, not only the Jäger and Segel model [JS], the Bellomo and Forni model $[\mathrm{BF}]$ and the models considered in $[\mathrm{ABL}]$, but also the models of Lachowicz and Wrzosek [LW1], as well as of Geigant, Ladizhansky and Mogilner [GLM].

In $[\mathrm{AGL}]$ and $[\mathrm{KKL}]$ various models of cellular tumour dynamics in competition with the immune system were proposed. The interacting individuals were the cells of the populations involved in the competition between the tumour and the immune system.

The models considered in [JS], [BF], [ABL], [GLM], [KKL], [LW1,2] and [La4] are stated in terms of systems of integro-differential equations (like the Boltzmann kinetic equation), with quadratic nonlinearities, describing the evolution of the densities of individuals.

The important problem is understanding relationships between different models developed at different levels of description. One can distinguish three possible levels of description as follows:

(I) the level of interacting individuals ("microscopic" description),

(II) the level of statistical description of test-individual (sometimes called a "mesoscopic" description),

(III) the "macroscopic" level.

In the context of kinetic theory of gases a huge bibliography on this topic exists (see references in [BGL], [La2,3,4], and [LM]), but the relationships between the different models are still not fully understood. The situation is definitely more complex in the case of biological systems.

Paper [La4] gives the conceptual framework for a program for finding possible transitions between the different levels of description with particular attention to the relationships between (II) to (III)).

The present paper is dedicated to the limits (I) to (II) and (I) to (III). Following the methods developed in kinetic theory [LP] it is shown that the appropiate systems composed of a large number $N$ of stochastic interacting indiviudals approximate the solution to Eq. (1.3). The control of rates of approximations by the parameters of the individuals system is provided. 
2. Generalized Kinetic Models. A general class of bilinear systems of Boltzmann-like integro-differential equations describing the dynamics of individuals undergoing kinetic (stochastic) interactions was proposed and analysed in [LW2]. These equations can model interactions between pairs of individuals of various populations at the level (II).

The class of equations in [LW2] can be regarded as a generalization of the Jäger and Segel kinetic model [JS], as well as those of Arlotti and Bellomo [AB1,2], Arlotti, Bellomo and Lachowicz [ABL], Lachowicz and Wrzosek [LW1], Geigant, Ladizhansky and Mogilner $[\mathrm{GLM}]$. In the literature these kind of models are referred to as the GKM, Generalized Kinetic Models.

The models refer to a (large) number of individuals of several populations: Each individual is characterized by the pair $\mathbf{u}=(j, u)$, where $j \in \mathcal{J}$ characterizes the population of the individual and $u \in \mathcal{U}$ is the (physical or biological) state (e.g. position, velocity, activation state, domination) of the individual, $\mathcal{J} \subset \mathbb{N}_{0}=\{0,1,2, \ldots\}$, and $\mathcal{U}$ is a domain in $\mathbb{R}^{d}, d \geq 1$. The evolution is determined by the interactions between pairs of individuals (analogously to kinetic theory only binary interactions are taken into account here).

The function $f=f(t, \mathbf{u}), f: \mathbb{R}_{+} \times \mathcal{J} \times \mathcal{U} \rightarrow \mathbb{R}_{+}, \mathbf{u}=(j, u)$, defines the density of individuals of the $j$-th population with state $u$ at time $t \geq 0$.

The total number of individuals at time $t \geq 0$ is given by

$$
\int_{\mathcal{J} \times \mathcal{U}} f(t, \mathbf{u}) \mathrm{d} \mu(\mathbf{u})
$$

where $\mu$ is a measure defined on $\mathcal{J} \times \mathcal{U}$ such that

$$
\mu\left(\mathcal{J}_{1} \times \mathcal{U}_{1}\right)=\delta\left(\mathcal{J}_{1}\right) \lambda\left(\mathcal{U}_{1}\right)
$$

$\delta$ is a (discrete) measure in $\mathcal{J}$ (e.g. the counting measure), $\mathcal{J}_{1}$ is a (finite) subset of $\mathcal{J}$, and $\lambda$ is the Lebesgue measure in $\mathcal{U}, \mathcal{U}_{1}$ is a $\lambda$-measurable subset of $\mathcal{U}$.

The rate of interaction between the individuals of the $j$-th population $(j \in \mathcal{J})$ with state $u \in \mathcal{U}$ and the individual of the $k$-th population $(k \in \mathcal{J})$ with state $v \in \mathcal{U}$ is given by the function

$$
a=a(\mathbf{u}, \mathbf{v}), \quad a:(\mathcal{J} \times \mathcal{U})^{2} \rightarrow \mathbb{R}_{+},
$$

where $\mathbf{u}=(j, u), \mathbf{v}=(k, v)$.

The transition into the $j$-th population $(j \in \mathcal{J})$ with state $u \in \mathcal{U}$ due to the interaction of individuals of the $k$-th population $(k \in \mathcal{J})$ with state $v \in \mathcal{U}$ with individuals of the $l$-th population $(l \in \mathcal{J})$ with state $w \in \mathcal{U}$ is described by the function

$$
A=A(\mathbf{u} ; \mathbf{v}, \mathbf{w}), \quad A:(\mathcal{J} \times \mathcal{U})^{3} \rightarrow \mathbb{R}_{+},
$$

where $\mathbf{u}=(j, u), \mathbf{v}=(k, v), \mathbf{w}=(l, w)$.

Referring to [LW2] the model (GKM) reads

$$
\partial_{t} f(t, \mathbf{u})=G[f](t, \mathbf{u})-f(t, \mathbf{u}) L f(t, \mathbf{u}), \quad \mathbf{u} \in \mathcal{J} \times \mathcal{U},
$$

where $G$ is the gain term,

$$
G[f](t, \mathbf{u})=\int_{(\mathcal{J} \times \mathcal{U})^{2}} A(\mathbf{u} ; \mathbf{v}, \mathbf{w}) a(\mathbf{v}, \mathbf{w}) f(t, \mathbf{v}) f(t, \mathbf{w}) \mathrm{d} \mu(\mathbf{v}) \mathrm{d} \mu(\mathbf{w}),
$$


and $f L f$ is the loss term,

$$
L f(t, \mathbf{u})=\int_{\mathcal{J} \times \mathcal{U}} a(\mathbf{u}, \mathbf{v}) f(t, \mathbf{v}) \mathrm{d} \mu(\mathbf{v}) .
$$

In the present paper the following particular (conservative) case is considered (cf. [La4]):

$$
\int_{\mathcal{J} \times \mathcal{U}} A(\mathbf{u} ; \mathbf{v}, \mathbf{w}) \mathrm{d} \mu(\mathbf{u})=1, \quad \text { for } \mu \text {-a.a. } \mathbf{v}, \mathbf{w} \text { in } \mathcal{J} \times \mathcal{U} .
$$

In this case the total number of individuals (2.2) is (formally) preserved and $A$ is a probability density with respect to the first variable.

Various models known in the literature can be covered by the above general model. In [LW2] and [La4] some examples were given. Here we mention

EXAmple 2.1. For some particular choices of functions $A$ and $a$ (constant with respect to $u, v$ and $w$ ) Eq. (2.3) results in an important model known in the literature, the Kermack-McKendrick ("SIR") model in theory of epidemics ([Ca]). Consider

$$
\mathcal{J}=\{1,2,3,4\}, \quad \mathcal{U} \text { such that } \lambda(\mathcal{U})=1,
$$

and $A(j, u ; k, v, l, w)$ and $a(j, u, k, v)$ independent of $u, v$ and $w$. Assume

$$
\begin{aligned}
& A(2 ; 1,2)=A(2 ; 2,1)=1, \quad A(i ; 1,2)=A(i ; 2,1)=0, \quad i=1,3,4, \\
& A(3 ; 2,4)=A(3 ; 4,2)=A(4 ; 2,4)=A(4 ; 4,2)=\frac{1}{2}, \\
& A(i ; 2,4)=A(i ; 4,2)=0, \quad i=1,2
\end{aligned}
$$

where $\gamma_{1}$ and $\gamma_{2}$ are given positive constants, while all other $a(j, k)$ are assumed to be equal 0. Hence Eq. (2.3) leads to the Kermack-McKendrick model

$$
\dot{f_{1}}=-\gamma_{1} \bar{f}_{1} \bar{f}_{2}, \quad \dot{\overline{f_{2}}}=\gamma_{1} \bar{f}_{1} \bar{f}_{2}-\gamma_{2} \bar{f}_{2}, \quad \dot{\bar{f}_{3}}=\gamma_{2} \bar{f}_{2},
$$

where $\bar{f}_{j}(t)=\int_{\mathcal{U}} f(t, j) \mathrm{d} \lambda(u)$, are densities of susceptibles, infectives and removed individuals, respectively for $j=1,2,3$, the constant density $\bar{f}_{4}$ plays an auxiliary rôle.

EXAMPLE 2.2. If $\mathcal{J}=\mathbb{N}=\{1,2,3, \ldots\}$ and

$$
A(j, u ; k, v, l, w)=\frac{1}{2} \delta_{j, k+l}, \quad a(j, u, k, v)=a_{j, k},
$$

where $a_{k, l}$ are given and independent of $u, v, w$, for $k, l \in \mathbb{N}$, then Eq. (2.3) is the Smoluchowski infinite system of equations describing the binary coagulation of colloids.

If $\mathcal{J}=\mathbb{N}$ and

$$
A(j, x ; k, y, l, z)=\frac{1}{2} \delta_{j, k+l} \tilde{A}_{k, l}(x ; y, z), \quad a(j, x, k, y)=a_{j, k}(x, y),
$$

where $\tilde{A}_{k, l}$ and $a_{k, l}$ are given (measurable) functions of $x, y, z$ and $x, y$, respectively, for $k, l \in \mathbb{N}$

$$
\int_{\mathcal{U}} \tilde{A}_{k, l}(x ; y, z) \mathrm{d} \lambda(x)=1 \quad \text { for } k, l \in \mathcal{J} \text { and } \lambda \text {-a.a. } y, z \text { in } \mathcal{U},
$$

then Eq. (2.3) is the nonlocal coagulation model proposed in [WL1]. Actually, in [WL1], the more general nonlocal coagulation-fragmentation model including diffusion according 
to Fick's law was proposed and analyzed. The variables $x$ and $y, z$ were interpreted as the positions (after and before the interaction, respectively) of interacting clusters in the physical space $\mathcal{U}$.

The model $S I R$ (2.7) refers to the conservative case. The conservativity of the coagulation models of Example 2.2 refers to the measure $\mu$ such that

$$
\delta\left(\mathcal{J}_{1}\right)=\sum_{j \in \mathcal{J}_{1}} j, \quad \mathcal{J}_{1} \subset \mathbb{N},
$$

that corresponds to the total mass conservation law in the coagulation process. This, however, is not directly expressed in terms of (2.4).

Example 2.3. Particular forms of Eq. (2.3) has been developed in order to describe the competition between a tumour and the immune system in [AGL] and [KKL]); the parameter $u \in \mathcal{U}=[0,1]$ is related to activation state of active tumour cells (in [AGL]) or tumour (leukemia) cells, macrophages, NK-cells and Tc-cells (in [KKL]). These models do not however correspond to the conservative case (2.4).

Some models that do not correspond to the conservative case (2.4) can result from Eq. (2.3).

ExAmple 2.4. Assume that $\mathcal{J}=\{1\}$ and $\mathcal{U} \subset \mathbb{R}^{1}$. Let $\alpha$ and $\beta$ be positive constants. Let $a(u, v)=\beta|v|$ and $A=A(u)$ be such that

$$
A \geq 0, \quad \int_{\mathcal{U}} A(u) \mathrm{d} \lambda(u)=1, \quad \int_{\mathcal{U}}|u| A(u) \mathrm{d} \lambda(u)=\frac{\alpha}{\beta} .
$$

Define

$$
\bar{f}=\int_{\mathcal{U}} f(u) \mathrm{d} \lambda(u), \quad \hat{f}=\int_{\mathcal{U}}|u| f(u) \mathrm{d} \lambda(u) .
$$

Consider the particular version of Eq. (2.3)

$$
\partial_{t} f(t, u)=\beta A(u) \bar{f}(t) \hat{f}(t)-\beta f(t, u) \hat{f}(t),
$$

with the initial data

$$
\left.f\right|_{t=0}=F, \quad \bar{F}=1 .
$$

By (2.12), Eq. (2.13) corresponds to the conservative case (2.4) and we have

$$
\bar{f}(t)=\bar{F}=1, \quad \text { for } t>0 .
$$

On the other hand, $\hat{f}$ satisfies the following logistic equation

$$
\frac{\mathrm{d}}{\mathrm{d} t} \hat{f}=\alpha \hat{f}-\beta \hat{f}^{2} .
$$

Similarly Eq. (2.3) can result in the competitive Verhulst-Volterra systems (cf. [La5]). The models (2.7) and (2.16) are macroscopic ones (the level (III)).

REMARK 2.1. Referring to the coagulation model described in Example 2.2 we consider $\mathcal{J}=\mathbb{N}, \mathcal{U}=[0,1]$, and

$$
\begin{gathered}
A(j, u ; k, v, l, w)=\delta_{j, k+l} A^{\prime}(u ; v, w), \\
a(j, u, k, v)=a_{j, k} v
\end{gathered}
$$


for all $j, k, l \in \mathcal{J}$ and $\lambda$-a.a. $u, v, w \in] 0,1[$;

$$
\begin{gathered}
\left.A^{\prime}(u ; v, w) \geq 0, \quad \lambda \text {-a.a. } u, v, w \in\right] 0,1[, \\
\left.\int_{0}^{1} A^{\prime}(u ; v, w) \mathrm{d} \lambda(u)=1, \quad \lambda \text {-a.a. } v, w \in\right] 0,1[,
\end{gathered}
$$

and $\left\{a_{j, k}\right\}_{(j, k) \in \mathcal{J}^{2}}$ are given nonnegative coagulation coefficients $a_{j, k}=a_{k, j}$. Therefore we are dealing with the conservative (2.4) case of the GKM (Eq. (2.3)). Moreover, assume that

$$
\left.\int_{0}^{1} u A^{\prime}(u ; v, w) \mathrm{d} \lambda(u)=\frac{v}{2}, \quad \lambda \text {-a.a. } v, w \in\right] 0,1[.
$$

Thus $\hat{f}_{j}(t)=\int_{0}^{1} u f(t, j, u) \mathrm{d} \lambda(u), j \in \mathcal{J}$, satisfy (formally) the Smoluchowski infinite coagulation system.

The analogous strategy can be applied for the nonlocal coagulation model [WL1].

The relationships between the particle systems and the Smoluchowski coagulation models were studied in a number of papers - see $[\mathrm{DF}],[\mathrm{DS}],[\mathrm{Gu}],[\mathrm{LX}]$ and references therein.

These examples together with those in [LW2] and [La4] show that Eq. (2.3) is a general structure which can be particularized in various important models. Paper [LW2] was a first step in the description of the mathematical properties of Eq. (2.3). It provides some existence and uniqueness theorems for Eq. (2.3), discusses its equilibrium solutions, and studies its diffusive limit. In [LW2] the existence of unstable equilibrium solutions which are inhomogeneous with respect to the $\mathbf{u}$-variable was proved. The case when only homogeneous equilibrium solutions exist was specified. Under suitable scaling it was proved that the one-dimensional version of Eq. (2.3) is asymptotically equivalent to the nonlinear porous medium equation also used in mathematical biology as the model for density dependent population dispersal. In [La4] research perspectives for finding possible transitions from (II) to (III) were presented. In the present paper the results on the relationships between (I) and (II) and then between (I) and (III) are proved.

3. Interaction of individuals. The important but still unsolved problem is derivation of kinetic equations ((II)) and then hydrodynamic equations ((III)) from particle dynamics $(\mathbf{I}))$. In view of the lack of a suitable mathematical theory allowing to derive first the kinetic and then the hydrodynamic equations from (deterministic) particle systems, the very important problem is to construct a theory starting from stochastic particle systems. On the other hand stochastic particle methods are widely used in the numerical simulations in kinetic theory (see [BB], [CPW], [PWZ], [Wa1,2], [Wl], and references therein).

The idea of approximating the Boltzmann equation by stochastic particle systems appeared in the 1930-ies in the paper by Leontovich [Le] but had rather been ignored by the mathematicians. In the spatially homogeneous case the derivation of a simplified Boltzmann-type equation (the so-called caricature of a Maxwellian gas) from a stochastic model was proposed by Kac [Ka]. Kac's model was studied by McKean [Mc]. Nowadays there is a huge literature on various stochastic approaches in the kinetic theory - see 
for example $[\mathrm{Ar}],[\mathrm{BB}],[\mathrm{CPW}],[\mathrm{GM}],[\mathrm{LP}],[\mathrm{La} 1],[\mathrm{PWZ}],[\mathrm{Sk}],[\mathrm{Wa} 1,2,3]$ and references therein.

In $[\mathrm{LP}]$ the starting point is a system of identical point particles that can interact in pairs, when the distance between two of them is not greater than some number (which is one of the parameters of the model). As a result of the interaction there are jumps of the velocities of two interacting particles. The main result of $[\mathrm{LP}]$ is related to approximations (in $L_{1}$ ) of both solution of the Boltzmann equation and macroscopic parameters given by a solution of the Euler system by the solution of the prospective equation (i.e. the modified Liouville equation) of the model. Approximations are realized if the parameters of the model are suitably chosen. All estimations of [LP] allow to control the rates of approximations. [La1] was a continuation of [LP]. In a sense, Skorohod's approach ([Sk]) which allows to construct processes in complex phase spaces by stochastic differential equations - was combined with derivation of the hydrodynamic equations from the kinetic equation.

Here we follow the idea of [LP]. We show that the solution of Eq. (1.3) can be approximated by solutions of (linear) equations describing the dynamics of a suitable system of interacting individuals.

Consider a system composed of $N$ interacting individuals. Every individual $n(n \in$ $\{1,2, \ldots, N\})$ is characterized by $\mathbf{u}_{n}=\left(j_{n}, u_{n}\right)$, where $j_{n} \in \mathcal{J}$ characterizes the population of the $n$-individual and $u_{n} \in \mathcal{U}$-its state. The $n$-individual interacts with the $m$-individual and the interaction take place at random times. After the interaction both individuals may change their population or/and their state.

Consider the Markov process of $N$ individuals with infinitesimal generator given by

$$
\begin{aligned}
& \Lambda_{N} \phi\left(\mathbf{u}_{1}, \mathbf{u}_{2}, \ldots, \mathbf{u}_{N}\right)=\frac{1}{N} \sum_{\substack{1 \leq n, m \leq N \\
n \neq m}} a\left(\mathbf{u}_{n}, \mathbf{u}_{m}\right) \\
& \quad \times\left(\int_{\mathcal{J} \times \mathcal{U}} A\left(\mathbf{v} ; \mathbf{u}_{n}, \mathbf{u}_{m}\right) \phi\left(\mathbf{u}_{1}, \ldots, \mathbf{u}_{n-1}, \mathbf{v}, \mathbf{u}_{n+1}, \ldots, \mathbf{u}_{N}\right) \mathrm{d} \mu(\mathbf{v})-\phi\left(\mathbf{u}_{1}, \ldots, \mathbf{u}_{N}\right)\right),
\end{aligned}
$$

where $\phi$ is an appropriate test function.

We are interested in the conservative case defined by (2.4) and therefore we assume that

$$
\int_{\mathcal{J} \times \mathcal{U}} A(\mathbf{u} ; \mathbf{v}, \mathbf{w}) \mathrm{d} \mu(\mathbf{u})=1, \quad \text { for } \mu \text {-a.a. } \mathbf{v}, \mathbf{w} \in \mathcal{J} \times \mathcal{U}
$$

Moreover, let

$$
0 \leq A(\mathbf{u} ; \mathbf{v}, \mathbf{w}), \quad 0 \leq a(\mathbf{u}, \mathbf{v}) \leq c_{a}, \quad \text { for } \mu \text {-a.a. } \mathbf{u}, \mathbf{v}, \mathbf{w} \in \mathcal{J} \times \mathcal{U},
$$

where $c_{A}$ and $c_{a}$ are positive constants.

Assume that the system is initially distributed according to the probability density $F^{N} \in L_{1}^{(N)}$, where $L_{1}^{(N)}$ is the space

$$
L_{1}^{(N)}=L_{1}\left(\mu^{N \otimes}\right)=L_{1}(\underbrace{\mu \otimes \ldots \otimes \mu}_{N \text { times }})
$$


equipped with the norm

$$
\|f\|_{L_{1}^{(N)}}=\int_{(\mathcal{J} \times \mathcal{U})^{N}}\left|f\left(\mathbf{u}_{1}, \ldots, \mathbf{u}_{N}\right)\right| \mathrm{d} \mu\left(\mathbf{u}_{1}\right) \ldots \mathrm{d} \mu\left(\mathbf{u}_{N}\right) .
$$

The time evolution is described by the probability density

$$
f^{N}(t)=\exp \left(t \Lambda_{N}^{*}\right) F^{N}
$$

It satisfies (in $L_{1}^{(N)}$ )

$$
\partial_{t} f^{N}=\Lambda_{N}^{*} f^{N} ;\left.\quad f^{N}\right|_{t=0}=F^{N}
$$

where

$$
\begin{aligned}
\Lambda_{N}^{*} f\left(\mathbf{u}_{1}, \mathbf{u}_{2}, \ldots, \mathbf{u}_{N}\right) & =\frac{1}{N} \sum_{\substack{1 \leq n, m \leq N \\
n \neq m}}\left(\int_{\mathcal{J} \times \mathcal{U}} A\left(\mathbf{u}_{n} ; \mathbf{v}, \mathbf{u}_{m}\right) a\left(\mathbf{v}, \mathbf{u}_{m}\right)\right. \\
& \left.\times f\left(\mathbf{u}_{1}, \ldots, \mathbf{u}_{n-1}, \mathbf{v}, \mathbf{u}_{n+1}, \ldots, \mathbf{u}_{N}\right) \mathrm{d} \mu(\mathbf{v})-a\left(\mathbf{u}_{n}, \mathbf{u}_{m}\right) f\left(\mathbf{u}_{1}, \ldots, \mathbf{u}_{N}\right)\right) .
\end{aligned}
$$

Under the assumptions (3.2) the operator $\Lambda_{N}^{*}$ is a bounded linear operator in the space $L_{1}^{(N)}$. Therefore the Cauchy Problem (3.4) has a unique solution (3.3) in $L_{1}^{(N)}$ for all $t \geq 0$. Moreover, by the standard argument we see that the solution is nonnegative for nonnegative initial data and the $L_{1}^{(N)}$-norm is conserved,

$$
\left\|f^{N}(t)\right\|_{L_{1}^{(N)}}=\left\|F^{N}\right\|_{L_{1}^{(N)}}=1, \quad \text { for } t>0 .
$$

We assume that all functions are symmetric,

$$
f^{N}\left(\mathbf{u}_{1}, \ldots, \mathbf{u}_{N}\right)=f^{N}\left(\mathbf{u}_{r_{1}}, \ldots, \mathbf{u}_{r_{N}}\right),
$$

for $\mu$-a.a. $\mathbf{u}_{1}, \ldots, \mathbf{u}_{N}$ in $\mathcal{J} \times \mathcal{U}$ and for any permutation $\left\{r_{1}, \ldots, r_{N}\right\}$ of the set $\{1, \ldots, N\}$.

We introduce the $s$-individual marginal density $(1 \leq s<N)$

$$
f^{N, s}\left(\mathbf{u}_{1}, \ldots, \mathbf{u}_{s}\right)=\int_{(\mathcal{J} \times \mathcal{U})^{N-s}} f^{N}\left(\mathbf{u}_{1}, \ldots, \mathbf{u}_{N}\right) \mathrm{d} \mu\left(\mathbf{u}_{s+1}\right) \ldots \mathrm{d} \mu\left(\mathbf{u}_{N}\right),
$$

and $f^{N, N}=f^{N}$.

The function $f^{N}$ satisfies Eq. (3.4) iff $f^{N, s}$ satisfy the following finite hierarchy of equations

$$
\partial_{t} f^{N, s}=\frac{s}{N} \Lambda_{s}^{*} f^{N, s}+\frac{N-s}{N} \Theta_{s+1} f^{N, s+1},
$$

for $s=1,2, \ldots, N$, where

$$
\begin{aligned}
\left(\Theta_{s+1} f\right)\left(\mathbf{u}_{1}, \ldots, \mathbf{u}_{s}\right)= & \sum_{n=1}^{s}\left(\int_{(\mathcal{J} \times \mathcal{U})^{2}} A\left(\mathbf{u}_{n} ; \mathbf{v}, \mathbf{w}\right) a(\mathbf{v}, \mathbf{w})\right. \\
& \times f\left(\mathbf{u}_{1}, \ldots, \mathbf{u}_{n-1}, \mathbf{v}, \mathbf{u}_{n+1}, \ldots, \mathbf{u}_{s}, \mathbf{w}\right) \mathrm{d} \mu(\mathbf{v}) \mathrm{d} \mu(\mathbf{w}) \\
& \left.-\int_{\mathcal{J} \times \mathcal{U}} a\left(\mathbf{u}_{n}, \mathbf{v}\right) f\left(\mathbf{u}_{1}, \ldots, \mathbf{u}_{s}, \mathbf{v}\right) \mathrm{d} \mu(\mathbf{v})\right)
\end{aligned}
$$

Taking $N$ sufficiently large we may expect that the solution of the finite hierarchy (3.7) approximates solution of the following infinite hierarchy of equations:

$$
\partial_{t} f^{s}=\Theta_{s+1} f^{s+1}, \quad s=1,2, \ldots
$$


The integral versions of hierarchies (3.7) and (3.8) read (3.9)

$$
f^{N, s}(t)=F^{N, s}+\frac{s}{N} \int_{0}^{t} \Lambda_{s} f^{N, s}\left(t_{1}\right) \mathrm{d} t_{1}+\frac{N-s}{N} \int_{0}^{t} \Theta_{s+1} f^{N, s+1}\left(t_{1}\right) \mathrm{d} t_{1}, \quad s=1, \ldots, N,
$$

and

$$
f^{s}(t)=F^{s}+\int_{0}^{t} \Theta_{s+1} f^{s+1}\left(t_{1}\right) \mathrm{d} t_{1}, \quad s=1,2, \ldots
$$

respectively.

Definition 3.1. An admissible hierarchy $\left\{f^{s}\right\}_{s=1,2,3, \ldots}$ is a sequence of functions $f^{s}$ satisfying (for $s=1,2, \ldots$ )

(i) $f^{s}$ is a probability density on $(\mathcal{J} \times \mathcal{U})^{s}$;

(ii) $f^{s}\left(\mathbf{u}_{1}, \ldots, \mathbf{u}_{s}\right)=f^{s}\left(\mathbf{u}_{r_{1}}, \ldots, \mathbf{u}_{r_{s}}\right)$ for $\mu$-a.a. $\mathbf{u}_{1}, \ldots, \mathbf{u}_{s}$ in $\mathcal{J} \times \mathcal{U}$ and for any permutation $\left\{r_{1}, \ldots, r_{s}\right\}$ of the set $\{1, \ldots, s\}$;

(iii) $f^{s}\left(\mathbf{u}_{1}, \ldots, \mathbf{u}_{s}\right)=\int_{\mathcal{J} \times \mathcal{U}} f^{s+1}\left(\mathbf{u}_{1}, \ldots, \mathbf{u}_{s+1}\right) \mathrm{d} \mu\left(\mathbf{u}_{s+1}\right)$ for $\mu$-a.a. $\mathbf{u}_{1}, \ldots, \mathbf{u}_{s}$ in $\mathcal{J} \times \mathcal{U}$.

We have

THEOREM 3.1. Let $\left\{F^{s}\right\}_{s=1,2, \ldots}$ be an admissible hierarchy. Then, for all $t>0$, there exists a unique hierarchy $\left\{f^{s}(t)\right\}_{s=1,2, \ldots}$ with $f^{s}(t) \in L_{1}^{(s)}(s=1,2, \ldots)$ which is a solution of Eq. (3.10) with initial data $f^{s}(0)=F^{s}(s=1,2, \ldots)$. Moreover $\left\{f^{s}(t)\right\}_{s=1,2, \ldots}$, for all $t>0$, is an admissible hierarchy.

Proof. By (3.1) and (3.2) we have

$$
\left\|\Theta_{s+1} f\right\|_{L_{1}^{(s)}} \leq c_{1} s\|f\|_{L_{1}^{(s+1)}}
$$

and

$$
\int_{(\mathcal{J} \times \mathcal{U})^{s}}\left(\Theta_{s+1} f\right)\left(\mathbf{u}_{1}, \ldots, \mathbf{u}_{s}\right) \mathrm{d} \mu\left(\mathbf{u}_{1}\right) \ldots \mathrm{d} \mu\left(\mathbf{u}_{s}\right)=0
$$

for all $f \in L_{1}^{(s+1)}$ and $s=1,2, \ldots$, where $c_{1}=2 c_{a}$ is a constant. Moreover,

$$
\int_{\mathcal{J} \times \mathcal{U}}\left(\Theta_{s+1} f\right)\left(\mathbf{u}_{1}, \ldots, \mathbf{u}_{s}\right) \mathrm{d} \mu\left(\mathbf{u}_{s}\right)=\left(\Theta_{s} \hat{f}\right)\left(\mathbf{u}_{1}, \ldots, \mathbf{u}_{s-1}\right)
$$

for all $f \in L_{1}^{(s+1)}$ and $s=1,2, \ldots$, where

$$
\hat{f}\left(\mathbf{u}_{1}, \ldots, \mathbf{u}_{s}\right)=\int_{\mathcal{J} \times \mathcal{U}} f\left(\mathbf{u}_{1}, \ldots, \mathbf{u}_{s-1}, \mathbf{u}_{s+1}, \mathbf{u}_{s}\right) \mathrm{d} \mu\left(\mathbf{u}_{s+1}\right) .
$$

Iterating Eq. (3.10) we obtain the following "perturbation series":

$$
f^{s}(t)=F^{s}+\sum_{m=1}^{\infty} \int_{0}^{t} \int_{0}^{t_{1}} \int_{0}^{t_{2}} \ldots \int_{0}^{t_{m-1}} \Theta_{s+1} \Theta_{s+2} \ldots \Theta_{s+m} F^{s+m} \mathrm{~d} t_{m} \ldots \mathrm{d} t_{1} .
$$

Existence of a solution, its uniqueness and representation in the form of (3.14) are directly obtained on the time interval $\left[0, t_{*}\right]$ on which the series, defined by the r.h.s. of (3.14), is convergent. 
The $L_{1}^{(s)}$-norm of the $m^{\text {th }}$-term of the series in the r.h.s. of (3.14) can be estimated by

$$
\left(c_{1} t\right)^{m} \frac{(s+m-1) !}{m !(s-1) !}
$$

Consequently, by

$$
\frac{s(s+1) \ldots(s+m-1)}{m !} \leq 2^{s+m-1},
$$

if $t_{*}=\frac{1}{4 c_{1}}$, then the series converges uniformly on $\left[0, t_{*}\right]$.

The hierarchy $\left\{f^{s}\right\}_{s=1,2, \ldots}$ satisfies, for all $\left.\left.t \in\right] 0, t_{*}\right]$ the property (ii), and by (3.13), the property (iii) of Definition 3.1.

The nonnegativity of the solution can be proved on a time interval $\left[0, t^{*}\right]$, where $0<t^{*} \leq t_{*}$, by standard arguments.

By (3.12) we have

$$
\left\|f^{s}(t)\right\|_{L_{1}^{(s)}}=\left\|F^{s}\right\|_{L_{1}^{(s)}}, \quad \text { for } 0 \leq t \leq t^{*}, \quad s=1,2, \ldots
$$

It follows that $\left\{f^{s}(t)\right\}_{s=1,2, \ldots}$ is an admissible hierarchy for all $t \in\left[0, t^{*}\right]$.

Assuming $\left\{f^{s}\left(t^{*}\right)\right\}_{s=1,2, \ldots}$ as initial datum, we can repeat the same arguments for $t \in\left[t^{*}, 2 t^{*}\right]$ and so on. This completes the proof of the theorem.

We assume now that the Markov process starts with chaotic (i.e. factorized) probability density and we consider the hierarchy (3.10) with initial data

$$
F^{s}=F \otimes \ldots \otimes F=(F)^{s \otimes}, \quad s=1,2, \ldots
$$

i.e. $s$-fold outer product of a probability density $F$ defined on $\mathcal{J} \times \mathcal{U}$. We may see that the propagation of chaos is held and the solution $f^{s}(t)$ to Eq. (3.10) is the $s$-product of solution $f(t)$ of Eq. (2.3). Therefore we have

Corollary 3.2. Let $F$ be a probability density on $\mathcal{J} \times \mathcal{U}$. Then, for each $t_{0}>0$, there exists an admissible hierarchy $\left\{f^{s}\right\}_{s=1,2, \ldots}$ such that

(i) it is a unique solution of Eq. (3.10) with chaotic initial data (3.17),

(ii) $f^{s}(t)$ is chaotic

$$
f^{s}(t)=(f(t))^{s \otimes},
$$

for all $0<t \leq t_{0}$ and $s=1,2, \ldots$, where $f(t)$ is the unique solution in $L_{1}^{(1)}$ of $E q$. (2.3) with the initial datum $F$.

We may now formulate the main result, namely the theorem stating that the solution of Eq. (2.3) is approximated by the solutions of Eq. (3.4) as $N \rightarrow \infty$ (the proof follows the line of $[\mathrm{LP}])$.

Theorem 3.3. Let $F$ be a probability density on $\mathcal{J} \times \mathcal{U}$. Then, for each $t_{0}>0$, there exists $N_{0}$ such that for $N \geq N_{0}$

$$
\sup _{\left[0, t_{0}\right]}\left\|f^{N, 1}-f\right\|_{L_{1}^{(1)}} \leq \frac{c_{2}}{N^{\eta}}
$$


where the nonnegative functions $f^{N, s} \in L_{1}^{(s)}(s=1, \ldots, N)$ form the unique solution of Eq. (3.9) corresponding to the initial datum

$$
f^{N, s}(0)=(F)^{s \otimes}, \quad s=1, \ldots, N \text {; }
$$

$f \in L_{1}^{(1)}$ is the unique, nonnegative solution of Eq. (2.3) corresponding to the initial datum $F ; \eta$ and $c_{2}$ are positive constants that depend on $t_{0}$.

Proof. Let $\left\{f^{N, s}\right\}_{s=1, \ldots, N}$ and $\left\{f^{s}\right\}_{s=1,2, \ldots}$ be the solutions of Eq. (3.9) and Eq. (3.10), respectively, corresponding to the initial data

$$
f^{N, s}(0)=f^{s}(0)=(F)^{s \otimes} .
$$

If $N<s$ we assume $f^{N, s} \equiv 0$.

We consider the difference

$$
\Delta^{N, s}(t)=f^{N, s}(t)-f^{s}(t), \quad t>0 .
$$

It satisfies, for $s=1, \ldots, N$ and $0 \leq t_{1}<t \leq t_{0}$, the following equation:

$$
\Delta^{N, s}(t)=\mathcal{G}^{N, s}\left(t, t_{1}\right)+\int_{t_{1}}^{t} \Theta_{s+1} \Delta^{N, s+1}\left(t_{2}\right) \mathrm{d} t_{2},
$$

where

$$
\mathcal{G}^{N, s}\left(t, t_{1}\right)=\Delta^{N, s}\left(t_{1}\right)+\frac{s}{N} \int_{t_{1}}^{t}\left(\Lambda_{s} f^{N, s}\left(t_{2}\right)-\Theta_{s+1} f^{N, s+1}\left(t_{2}\right)\right) \mathrm{d} t_{2} .
$$

Let $\tau_{k}=t^{*} k$, where $t^{*}=\frac{1}{8 c_{1}}, c_{1}$ is defined in $(3.11)$, and $k=1,2, \ldots,\left[\frac{t_{0}}{t}\right]+1$. Set

$$
a_{k}^{N, s}=\sup _{\left[\tau_{k-1}, \tau_{k}\right]}\left\|\Delta^{N, s}\right\|_{L_{1}^{(s)}} .
$$

We want to prove

$$
a_{k}^{N, s} \leq \alpha_{k}^{N}
$$

for $s$ such that

$$
2^{s} \leq N^{\frac{\eta_{k}}{2}}
$$

where $\alpha_{k}^{N}=2^{5 k} \frac{1}{N^{\eta_{k}}}$ and $\eta_{k}=\frac{1}{2^{2 k+2}}$. We have

$$
\left\|\mathcal{G}^{N, s}\left(t, \tau_{k-1}\right)\right\|_{L_{1}^{(s)}} \leq\left\|\Delta^{N, s}\left(\tau_{k-1}\right)\right\|_{L_{1}^{(s)}}+2 c_{1} t^{*} \frac{s^{2}}{N}
$$

and

$$
\left\|\Delta^{N, s}(t)\right\|_{L_{1}^{(s)}} \leq\left\|\mathcal{G}^{N, s}\left(t, \tau_{k-1}\right)\right\|_{L_{1}^{(s)}}+c_{1} s \int_{\tau_{k-1}}^{t}\left\|\Delta^{N, s+1}\left(t_{2}\right)\right\|_{L_{1}^{(s+1)}} \mathrm{d} t_{2},
$$

for $t \in\left[\tau_{k-1}, \tau_{k}\right]$. If (3.24) is satisfied for $k-1$ then by (3.27) we obtain

$$
\left\|\mathcal{G}^{N, s}\left(t, \tau_{k-1}\right)\right\|_{L_{1}^{(s)}} \leq \alpha_{k-1}^{N}+\frac{s^{2}}{N} \text {. }
$$

If moreover $N$ is sufficiently large then by (3.26)

$$
\left\|\mathcal{G}^{N, s}\left(t, \tau_{k-1}\right)\right\|_{L_{1}^{(s)}} \leq 2 \alpha_{k-1}^{N} .
$$

Using (3.30) we may iterate (3.28) up to the largest $m$ for which

$$
2^{s+m} \leq N^{\frac{\eta_{k-1}}{2}}
$$


and assume that

$$
2^{m} \geq \frac{1}{2^{5 k-1}} N^{\frac{\eta_{k-1}-\eta_{k}}{2}}
$$

We have

$$
\begin{aligned}
\left\|\Delta^{N, s}(t)\right\|_{L_{1}^{(s)}} \leq & 2 \alpha_{k-1}^{N}\left(1+\sum_{l=1}^{m-1} \frac{s(s+1) \ldots(s+l-1)}{4^{l} l !}\right) \\
& +2 \frac{s(s+1) \ldots(s+m-1)}{4^{m} m !}
\end{aligned}
$$

for $t \in\left[\tau_{k-1}, \tau_{k}\right]$, where the last term on the r.h.s. of (3.33) is obtained by using the obvious estimate

$$
\left\|\Delta^{N, s}\right\|_{L_{1}^{(s)}} \leq 2
$$

By (3.33) and (3.15) it follows that

$$
a_{k}^{N, s} \leq 2^{s+1} \alpha_{k-1}^{N}+2^{s-m} .
$$

Now the proof of (3.25) follows by induction on $k$. We first show that (3.25) is satisfied for $k=1$ and then assume that it holds for $k-1 \geq 1$. Using (3.26), (3.32) and (3.34) we obtain

$$
a_{k}^{N, s} \leq 2^{5 k-4} N^{\frac{\eta_{k}}{2}-\eta_{k-1}}+2^{5 k-1} N^{\eta_{k}-\frac{\eta_{k-1}}{2}}
$$

and therefore (3.25) for every $k$ follows.

Theorem 3.2 shows that the solution of the Boltzmann-like bilinear integro-differential system of equations can be approximated by the solutions of linear equations describing the stochastic system of individuals - provided that the parameters of the stochastic system are suitably chosen.

The estimates are not optimized. One can hope that some of them can be improved to make them uniform with respect to $t_{0}$.

\section{References}

[AB1] L. Arlotti and N. Bellomo, Population dynamics with stochastic interaction, Transport Theory Statist. Phys. 24 (1995), 431-443.

[AB2] L. Arlotti and N. Bellomo, Solution of a new class of nonlinear kinetic models of population dynamics, Appl. Math. Lett. 9 (1996), 65-70.

[ABL] L. Arlotti, N. Bellomo and M. Lachowicz, Kinetic equations modelling population dynamics, Transport Theory Statist. Phys. 29 (2000), 125-139.

[AdB] J. A. Adam and N. Bellomo (eds.), A Survey of Models for Tumor-Immune System Dynamics, Birkhäuser, Boston, 1997.

[AGL] L. Arlotti, A. Gamba and M. Lachowicz, A kinetic model of tumour/immune system cellular interactions, J. Theoret. Medicine 4 (2002), 39-50.

[AL] L. Arlotti and M. Lachowicz, Qualitative analysis of a nonlinear integro-differential equation modelling tumor-host dynamics, Math. Comput. Modelling 23 (1996), 11-29.

[Ar] A.A. Arsen'ev, Approximation of the Boltzmann equation by stochastic equations, Zh. Vychisl. Mat. i Mat. Fiz. 28 (1988), 560-567 (in Russian). 
[BB] H. Babovsky and R. Illner, A convergence proof for Nanbu's simulation method for the full Boltzmann equation, SIAM J. Numer. Anal. 26 (1989), 45-65.

[BC] J. M. Ball and J. Carr, The discrete coagulation-fragmentation equations: existence, uniqueness and density conservation, J. Statist. Phys. 61 (1990), 203-234.

[BD] N. Bellomo and E. De Angelis, Strategies of applied mathematics towards an immunomathematical theory on tumours and immune system interactions, Math. Models Methods Appl. Sci. 8 (1998), 1403-1429.

[BF] N. Bellomo and G. Forni, Dynamics of tumor interaction with the host immune system, Math. Comput. Modelling 20 (1994), 107-122.

[BGL] C. Bardos, F. Golse and D. Levermore, Fluid dynamics limits of kinetic equations II, Comm. Pure Appl. Math. 46 (1993), 667-753.

[Ca] V. Capasso, Mathematical Structures of Epidemic System, Springer, New York 1996.

[CPW] S. Caprino, M. Pulvirenti and W. Wagner, Stationary particle systems approximating stationary solutions to the Boltzmann equation, SIAM J. Math. Anal. 29 (1998), 913934.

[DF] M. Deaconu and N. Fournier, Probabilistic approach of some discrete and continuous coagulation equation with diffusion, Stochastic Process. Appl. 101 (2002), 83-111.

[DS] P. Donnelly and S. Simons, On the stochastic approach to cluster size distribution during particle coagulation, J. Phys. A: Math. Gen. 26 (1993), 2755-2767.

[GLM] E. Geigant, K. Ladizhansky and A. Mogilner, An intergrodifferential model for orientational distribution of F-Actin in cells, SIAM J. Appl. Math. 59 (1998), 787-809.

[GM] C. Graham and S. Méléard, Stochastic particle approximations for generalized Boltzmann models and convergence estimates, Ann. Probab. 25 (1997), 115-132.

[Gu] F. Guias, Coagulation-fragmentation processes: relations between finite particle models and differential equations, Preprint 98-41 (SFB 359), Juli 1998, Heidelberg.

[He] R. B. Herberman, NK Cells and Other Natural Effector Cells, Academic Press, New York, 1982.

[JS] E. Jäger and L. Segel, On the distribution of dominance in a population of interacting anonymous organisms, SIAM J. Appl. Math. 52 (1992), 1442-1468.

[Ka] M. Kac, Probability and Related Topics in Physical Sciences, Wiley-Interscience, New York, 1959.

[KKL] M. Kolev, E. Kozłowska and M. Lachowicz, A mathematical model for a single cell cancer-immune system dynamics, Math. Comput. Modeling, to appear.

[La1] M. Lachowicz, A system of stochastic differential equations modeling the Euler and the Navier-Stokes hydrodynamic equations, Japan J. Industr. Appl. Math. 10 (1993), 109-131.

[La2] M. Lachowicz, Asymptotic analysis of nonlinear kinetic equations: The hydrodynamic limit, in: Lecture Notes on the Mathematical Theory of the Boltzmann Equation, N. Bellomo (ed.), World Sci., Singapore, 1995, 65-148.

[La3] M. Lachowicz, Hydrodynamic limits of some kinetic equations, in: Mathematical Analysis in Fluid and Gas Dynamics, A. Matsumura and S. Kawashima (eds.), RIMS Kokyuroku 1146, Kyoto, 2000, 121-142.

[La4] M. Lachowicz, From microscopic to macroscopic description for generalized kinetic models, Math. Models Methods Appl. Sci. 12 ( 2002), 985-1005.

[La5] M. Lachowicz, Describing competitive systems at the level of interacting individuals, in: Proc. of the Eighth Nat. Confer. Appl. Math. Biol. Medicine (Łajs, 2002), 95-100.

[Le] M. A. Leontovich, Fundamental equations of the kinetic theory of gases from the point of view of stochastic processes, Zhur. Exper. Teoret. Fiz. 5 (1935), 211-231 (in Russian). 
[LM] P.-L. Lions and N. Masmoudi, From the Boltzmann equation to the equations of incompressible fluid mechanics, Arch. Rational Mech. Anal. 158 (2001), 173-211.

[LP] M. Lachowicz and M. Pulvirenti, A stochastic particle system modeling the Euler equation, Arch. Rational Mech. Anal. 109 (1990), 81-93.

[LW1] M. Lachowicz and D. Wrzosek, A nonlocal coagulation-fragmentation model, Appl. Math. (Warsaw) 27 (2000), 45-66.

[LW2] M. Lachowicz and D. Wrzosek, Nonlocal bilinear equations. Equilibrium solutions and diffusive limit, Math. Models Methods Appl. Sci. 11 (2001), 1375-1390.

[LX] R. Lang and N. Xanh, Smoluchowski's theory of coagulation in colloids holds rigorously in the Boltzmann-Grad limit, Z. Wahrsch. Verw. Geb. 54 (1980), 227-280.

[Mc] H. P. McKean, Speed of approach to equilibrium for Kac's caricature of maxwellian gas, Arch. Rational Mech. Anal. 21 (1966), 347-367.

[ODA] H. G. Othmer, S. R. Dunbar and W. Alt, Models of dispersal in biological systems, J. Math. Biol. 26 (1988), 263-298.

[PWZ] M. Pulvirenti, W. Wagner and M.B. Zavelani Rossi, Convergence of particle schemes for the Boltzmann equation, European J. Mech. B Fluids 13 (1994), 339-351.

[Sk] A. V. Skorohod, Stochastic Equations for Complex Systems, Nauka, Moscow, 1983 (in Russian) and Reidel, Dordrecht, 1988.

[Sm] M. Smoluchowski, Versuch einer mathematischen Theorie der kolloiden Lösungen, Z. Phys. Chem. 92 (1917), 129-168.

[Sz] A. S. Sznitman, Topics in propagation of chaos, in: Lecture Notes in Math. 1464, Springer, Berlin, 1991, 165-251.

[Wa1] W. Wagner, A stochastic particle system associated with the spatially inhomogeneous Boltzmann equation, Transport Theory Statist. Phys. 23 (1994), 455-478.

[Wa2] W. Wagner, A convergence proof for Bird's direct simulation Monte Carlo method for the Boltzmann equation, J. Statist. Phys. 66 (1992), 455-478.

[Wa3] W. Wagner, A functional law of large numbers for Boltzmann type stochastic particle systems, Stochastic Anal. Appl. 14 (1996), 591-636.

[Wl] W. Waluś, Computational methods for the Boltzmann equation, in: Lecture Notes on the Mathematical Theory of the Boltzmann equation, N. Bellomo (ed.), World Sci., Singapore, 1995, 179-223. 